\title{
ESTABLISHING AND MAINTAINING AN FRC AT BIRTH
}

\section{S.B. Hooper}

\section{Monash Institute for Medical Research, Monash University, Clayton, VIC, Australia}

The transition to life after birth is dependent upon airway liquid clearance by allowing pulmonary gas exchange to begin. This initiates major changes in cardiovascular physiology that allows the infant to successfully adapt to life after birth. As lung aeration is critical for the transition to air-breathing at birth, it is essential to understand the mechanisms involved to develop procedures that facilitate these processes in very preterm infants.

We have used phase contrast X-ray imaging to image air entry into lungs at birth and have investigated ventilation procedures that facilitate uniform lung aeration and prevent lung collapse in very preterm rabbits. Images acquired during spontaneous breathing demonstrate that lung aeration is mainly driven by transpulmonary pressure gradients, which provides a new understanding of how aeration can be facilitated in preterm infants. An initial sustained inflation (SI; $20 \mathrm{secs}$ ) over comes the long time constant required to move liquid through the airways and fully recruit FRC and tidal volume before the onset of tidal breathing. It is currently unclear if multiple shorter ( 3 secs) SIs are as effective as a single long SI and, if not, how do we know when the lung has successfully aerated. Furthermore, we have investigated the role of additional factors, such as the rate of expiratory gas flow, on the maintenance of FRC after birth.

These studies are clarifying the primary factors regulating airway liquid clearance and lung aeration at birth and provide a new understanding of how this can be facilitated in preterm infants. 